\title{
Head and Neck Cancer - New Insights into a Heterogeneous Disease
}

\author{
Jens Peter Klussmann \\ Department of Otorhinolaryngology, Head and Neck Surgery, University of Gießen, Gießen, Germany
}

The large majority of head and neck cancers are squamous cell carcinomas (HNSCC) that arise at the mucosal linings of the upper aerodigestive tract, suggesting a homogeneous disease. However, HNSCC are remarkably heterogeneous. They include several subclassifications in relation to anatomic location, etiology, and molecular findings. In Germany, 4,532 and 12,992 new HNSCC cases in women and men, respectively, were counted in 2013. These HNSCC developed at different locations, i.e., oral cavity, pharynx, and larynx [1]. The 5-year overall survival for all locations is still poor and is estimated at $51 \%$ for men and $61 \%$ for women in Germany. Historically, HNSCC have been linked to tobacco and alcohol consumption. Over the past decades, the overall incidence of HNSCC has been decreasing. In contrast to that, the incidence of oropharyngeal cancers is rising, which is related to a strong increase of human papillomavirus(HPV)-associated cancers in western countries. The reason for this increase might be a sexual transmission of HPV decades before cancer development. These cancers are now identified as a distinct tumor entity, with many differences in comparison to HPV-negative HNSCC regarding their cellular, biological, and clinical characteristics. The identification of HPVrelated HNSCC by molecular biology and recently omics approaches has disclosed the heterogeneity of HNSCC and thus has contributed to a new classification of these tumors. Strikingly, it became evident that HPV-related oropharyngeal cancers showed a significantly better response to established treatment modalities resulting in an improved overall survival. Therefore, the HPV status can be regarded as one of the most accurate prognostic biomarkers in HNSCC, and p16-immunostaining became a reliable surrogate marker for the HPV status. In line with this, the latest version of the 'AJCC Cancer Staging Manual' [2] includes new staging rules for HPV-positive cancer.
Despite improvements in diagnosis and therapy, morbidity and mortality have remained high and appropriate treatment of patients still is a major challenge. Most cases are diagnosed with locally advanced disease, and multimodal treatment consists of concomitant chemoradiotherapy or surgery followed by riskadapted radio(chemo)therapy. Although it is conceivable that distinct patient subgroups might benefit from treatment de-escalation (e.g., HPV-related HNSCC) or targeted therapy, effective personalized treatment options are not yet in clinical use for HNSCC.

The aim of this issue of Oncology Research and TreatMENT is to highlight new insights into the biology of HNSCC, to discuss their possible implications for established treatment modalities, to point to future therapeutic developments, and to call attention to open questions which should be addressed by clinical trials. Jou and Hess [3] focused on recent data derived from integrative genomics analysis and multi-scale modeling approaches regarding the biological and clinical diversity of HNSCC. Wagner et al. [4] gave an overview of the newly recognized tumor entity 'HPV-related HNSCC'. Semrau [5] discussed modern treatment technologies and new developments of radiation oncology. In their original article, Wuerdemann et al. [6] compared the outcome of patient cohorts with HPV-related and -unrelated oropharyngeal cancers treated with primary surgery. Schuler at al. [7] outlined novel treatment options for HNSCC, especially in the field of immunotherapy.

\section{Disclosure Statement}

The author declares no conflict of interest.

\section{KARGER}

(c) 2017 S. Karger GmbH, Freiburg

Fax +497614520714
Univ.-Prof. Dr. med. Jens Peter Klussmann 


\section{References}

1 Robert Koch-Institut: Bericht zum Krebsgeschehen in Deutschland 2016. Berlin, Robert Koch-Institut, 2016.

2 Amin MB, Edge SB, Greene FL, et al (eds): AJCC Cancer Staging Manual, ed 8. New York, Springer, 2017.

3 Jou A, Hess J: Epidemiology and molecular biology of head and neck cancer. Oncol Res Treat 2017;40:DOI 10.1159/000477127.
4 Wagner S, Sharma S, Wuerdemann N, et al: Human papillomavirus-related head and neck cancer. Oncol Res Treat 2017;40:DOI: 10.1159/000477252.

5 Semrau R: The role of radiotherapy in the definitive and postoperative treatment of advanced head and neck cancer. Oncol Res Treat 2017;40:DOI: 10.1159/ 000477128.
6 Wuerdemann N, Wittekindt C, Sharma S, et al: Risk factors for overall survival outcome in surgically treated human papillomavirus-negative and -positive patients with oropharyngeal cancer. Oncol Res Treat 2017;40:DOI: 10.1159/000477097.

7 Schuler P, Laban S, Doescher J, et al: Novel treatment options in head and neck cancer. Oncol Res Treat 2017;40:DOI: 10.1159/000477254. 